\title{
Pigment retinopathy in warmblood horses with equine degenerative myeloencephalopathy and equine motor neuron disease
}

\author{
Carrie J. Finno ${ }^{\star}$, Heather J. Kaese ${ }^{\dagger}$, Andrew D. Miller ${ }^{\ddagger}$, Giuliana Gianino*, Thomas Divers§, \\ and Stephanie J. Valbergף \\ "Population Health and Reproduction, School of Veterinary Medicine, School of Medicine, \\ University of California, Davis, CA 95616, USA \\ †Eye Care for Animals, Lees Summit, MO 64064, USA \\ ¥Section of Anatomic Pathology, Department of Biomedical Sciences Medicine, Cornell University \\ College of Veterinary, Ithaca, NY 14853, USA \\ §Department of Clinical Sciences, Cornell University College of Veterinary Medicine, Ithaca, NY \\ 14853, USA \\ "Large Animal Clinical Sciences, College of Veterinary Medicine, Michigan State University, East \\ Lansing, MI 48895, USA
}

\begin{abstract}
Objective-A pigment retinopathy has been reported in adult horses with equine motor neuron disease (EMND) arising from chronic a-tocopherol ( $a-T P)$ deficiency. A pigment retinopathy has not been identified in horses with neuroaxonal dystrophy/equine degenerative myeloencephalopathy (NAD/EDM) that affects genetically susceptible young horses with a-TP deficiency. The objective of this report is to describe, for the first time, a pigment retinopathy in a family of a-TP-deficient Warmbloods (WB) with clinically apparent NAD/EDM or EMND.
\end{abstract}

Animals and Procedures-Twenty-five WB horses from one farm underwent complete neurologic and ophthalmic examinations and serum a-TP concentrations were assessed. Two of the most severely ataxic horses were euthanized and postmortem examinations performed.

Results-Alpha-TP deficiency was widespread on this farm ( 22 of 25 horses). Eleven of 25 horses were clinically normal (age range 2-12 years), one had signs of EMND (6 years of age), 10 had signs of ataxia consistent with NAD/EDM (1-10 years), and two of these were postmortem confirmed concurrent NAD/EDM and EMND. A pigment retinopathy characterized by varying amounts of granular dark pigment in the tapetal retina was observed in four clinically apparent NAD/EDM horses (two postmortem confirmed concurrent NAD/EDM and EMND) and one horse with clinical signs of EMND.

Address communications to: C.J. Finno, Tel: 530 752-2739, Fax: (530) 752-9815, cjfinno@ucdavis.edu. 
Conclusions-A pigment retinopathy can be present in young a-TP-deficient Warmblood horses with clinical signs of EMND as well as those with signs of NAD/EDM.

\section{Keywords}

horse; lipofuscin; neurodegeneration; retina; tocopherol; vitamin E

\section{INTRODUCTION}

Vitamin E (vitE) describes a family of eight different fat-soluble naturally occurring closely related compounds. ${ }^{1}$ The family consists of two subgroups: tocopherols (saturated) and tocotrienols (unsaturated). Within each subgroup, there are four individual isoforms ( $a, \beta, \gamma$, and $\delta$ ). Alpha-tocopherol (a-TP), specifically the RRR stereoisomer (RRR-a-TP), is the most bioavailable isoform due to preferential uptake by the liver. ${ }^{2}$ Alpha-TP is the isoform that is used to assess whole body vitE status, with normal equine serum concentrations $>2$ $\mu \mathrm{g} / \mathrm{mL} .^{2}$ Deficiencies in vitE, specifically a-TP, have been associated with pigment retinopathies across species. ${ }^{3-9}$ The photoreceptor outer segments of the retina are particularly vulnerable to a deficiency of vitE due to the plentiful supply of oxygen, abundant mitochondria and a high rate of oxidative metabolism, resulting in increased free radical species and lipid peroxidation.

Whether or not a deficiency of vitE has an impact on neuronal health in a particular individual depends upon genetic factors, the temporal occurrence of deficiency during the development or duration of deficiency during adulthood. ${ }^{10}$ In the horse, neuroaxonal dystrophy/equine degenerative myeloencephalopathy (NAD/EDM) is a neurodegenerative disease characterized by a general proprioceptive ataxia that affects genetically susceptible foals if they are deficient in a-TP during the first year of life. ${ }^{11-13}$ Equine motor neuron disease (EMND) results in clinical signs of lower motor neuron weakness in older horses that have been $\mathrm{a}$-TP deficient for $\geq 18$ months and with no genetic predisposition. ${ }^{14}$ In horses with EMND, lipofuscin deposits are often observed on fundic examination and flash electroretinogram (ERG) of EMND-affected horses has demonstrated decreased B-wave amplitudes. ${ }^{9}$ We have previously reported no evidence of retinal pathology or ERG changes in a population of QHs with NAD/EDM. ${ }^{15}$ The purpose of this report is to describe for the first time a pigment retinopathy among a family of 25 related Warmblood (WB) horses that were associated with a-TP deficiency and signs of EMND or NAD/EDM.

\section{MATERIALS AND METHODS}

\section{Horses}

Twenty-five WB horses (Hanoverian, Trakehner, Dutch and Oldenburg; 12 geldings, 1 stallion, and 12 mares), ranging in age from 1 to 21 years (mean $6.5 \pm 4.5$ years) underwent a full neurologic examination by two examiners (CJF, SJV) and complete ophthalmic examination by one examiner (HJK). All neurologic examinations were video-recorded, and five-generation pedigrees were obtained. A full pedigree of all horses was constructed using pedigraph. ${ }^{16}$ Blood was collected for DNA and serum a-TP concentrations. ${ }^{11}$ All 
examinations and collections were performed in May. Horses were maintained on grass hay with no access to pasture.

\section{Serum a-TP concentrations}

High-performance liquid chromatography with fluorescence detection was used as previously described ${ }^{11}$ to measure serum $\mathrm{a}$-TP in serum samples that had been protected from light and maintained on ice. Serum samples were obtained prior to any supplementation with $\mathrm{a}$-TP for all but one horse that did not have a presupplementation sample available to assess (WB4). The referring veterinarian obtained eight presupplementation samples and a-TP was re-evaluated all 25 horses at the time of examination (Table 1).

One of three formulations (program A, B, or C) of a-TP had been administered to 8 of 25 examined horses in the 60 days preceding examination (Table 1). Supplementation program A (WB7, WB15, and WB22) consisted of 750 IU of RRR-a-TP q24h 6 days/week, program B (WB3, WB4, WB5) consisted of 8400 IU synthetic (dl-) a-tocopheryl acetate plus 400 IU RRR-a-tocopheryl acetate q24h 7 days/week, and program C (WB1, WB24) consisted of 4800 IU of synthetic (dl-) a-tocopheryl acetate q24h 7 days/week.

\section{Neurologic examination}

A complete neurologic assessment, including evaluation of cranial nerves, mentation, muscle mass and symmetry, cervicofacial and cutaneous trunci reflexes, tail and anal tone, stance and a full dynamic assessment with each horse circled, backed, walked up and down an incline, walked with the head elevated, and a tail pull test, was performed. Each independent examiner assigned a clinical neurologic score to each horse, classified by the type of ataxia if present (general proprioceptive, cerebellar, and vestibular) or if there were clinical signs attributable to lower motor neuron disease, as previously described. ${ }^{17}$ Both examiners were blinded to the a-TP status of the horses.

\section{Ophthalmic examination}

About $1 \%$ tropicamide (Tropicacyl ${ }^{\circledR}$; Cardinal Health, Dublin, OH, USA) was applied to each eye 15 min prior to examination. Horses were then sedated with xylazine (Rompun ${ }^{\circledR}$; Mobay Corporation, Animal Health Division, Shawnee, KS, USA) $(0.2 \mathrm{mg} / \mathrm{kg})$ intravenously. A complete ophthalmic examination was performed using a slit-lamp bimicroscope and an indirect ophthalmoscope by a board-certified ophthalmologist (HJK) and images obtained using the Clear View Optical Imaging System (Optibrand, Ft. Collins, $\mathrm{CO}$, USA). The ophthalmologist was masked to the results of the neurologic evaluation. Based on the fundic lesions, the degree of pigment retinopathy was classified as 'severe' (large amount of pigment accumulation), 'moderate' (moderate amount of pigment accumulation), 'mild' (mild amount of pigment accumulation), or 'absent' (no notable findings or incidental findings).

\section{Postmortem examination}

Two of the most severely affected horses (WB1 and WB2) were subsequently euthanized, and a full postmortem examination was performed (ADM). The entire nervous system was 
systematically examined, including the brain, spinal cord, peripheral nerves, and skeletal muscle.

\section{RESULTS}

\section{Serum a-TP concentrations}

Presupplementation a-TP concentrations were below the reference range in 22 of 24 horses for which values were available [seven of eight values from the referring veterinarian, 15 of 16 unsupplemented horses sampled at the time of authors' examination; one horse (WB4) only had a postsupplementation sample available and one horse had a normal presupplementation a-TP concentration (WB15), Table 1]. Mean a-TP concentrations increased from a mean of 1.08 to $2.66 \mu \mathrm{g} / \mathrm{mL}$ (reference range $2-4 \mu \mathrm{g} / \mathrm{mL}^{2}$ ) in horses that received any form of a-TP supplementation. At the time of examination, 17 of 25 horses, including one horse that had been previously receiving a-TP supplementation through program ' $\mathrm{C}$ ', were deficient in a-TP. There was no significant difference in presupplementation a-TP concentrations between suspect NAD/EDM horses and neurologically normal horses $(P=0.75$; Table 1$)$.

\section{Neurologic examination}

Behavior, mentation, and cranial nerve function were normal in all horses. One horse had an inconsistent menace response in OS (WB15; grade ataxia two of five). One horse (WB3) demonstrated symmetric muscle atrophy and lower motor neuron weakness, strongly resembling EMND. ${ }^{17}$ The owner declined additional diagnostics, including a peripheral nerve or muscle biopsy evaluation to confirm this diagnosis. The most consistent neurologic abnormality was a general proprioceptive ataxia observed in 15 of 25 horses, with scores ranging from 1 to 4 as previously described. ${ }^{11}$ Based upon our previous genetic studies using a mean cut-off general proprioceptive ataxia score of 2, ${ }^{18,19} 10$ of 25 horses were classified as suspect NAD/EDM cases, 5 of 25 as equivocal, 9 of 25 as normal, and one horse diagnosed with EMND. An abnormal cutaneous trunci reflex was apparent in 7 of 25 horses (bilaterally in five and unilaterally in two). Of these seven horses, five had a general proprioceptive score of $\geq 2$, one had a score of 1 of 5 (equivocal) and one 0 of 5 .

\section{Ophthalmic examination}

Visual deficits were not appreciated based on menace responses and based on the horses' reactions during the neurologic examination. The fundic examination of the horse with clinical signs of EMND revealed a severe pigment retinopathy (WB3; Fig. 1). Of the 10 horses with a clinical diagnosis of NAD/EDM, two horses had a moderate degree of pigment retinopathy (Fig. 2), two were classified as mild, and six had no pigment retinopathy observed (Table 1). Of the nine horses classified as neurologically normal, all had no evidence of pigment retinopathy. Additional findings that were considered incidental or a normal variation included prominent choroidal vessels in the 10 of 25 horses, tapetal hypoplasia (WB22 and WB15), and an inactive chorioretinal lesion (WB14). 


\section{Postmortem examination}

Within the cuneate nucleus of the medulla from both WB1 and WB2, there were small numbers of chromatolytic neurons and spheroids, a decrease in neuronal density and a subjective increase in glial cells. Within the cervical and thoracic spinal cord segments, scattered chromatolytic neurons and spheroids with focal regions of lipofuscin were noted in the ventral gray column of both horses. Regions of myelin vacuolation were observed in the ventral funiculi. The lesions within the cuneate nucleus and ventral funiculi are consistent with a diagnosis of NAD/EDM in both WB1 and WB2. ${ }^{11}$ Chromatolytic neurons in the ventral gray column of the spinal cord is typically associated with EMND. ${ }^{20}$ Therefore, while these horses clinically resembled cases of NAD/EDM, histologic lesions supportive of both NAD/EDM and EMND were observed. No significant findings were evident on histologic evaluation of both eyes from the two horses; however, the sections were suboptimal due to processing artifacts.

\section{Pedigree analysis}

The two postmortem confirmed concurrent NAD/EDM and EMND geldings were full siblings (Fig. 3; WB1 and WB2) and, similar to four other clinically apparent NAD/EDM cases, were sired by WB20 (grade 1 of 5 or equivocal general proprioceptive ataxia). The clinically affected EMND horse (WB3) was also by the same sire (WB20). One NAD/EDM clinically affected mare (WB15) was sired by a NAD/EDM clinically affected stallion (WB23; grade 2 of 5 ataxia). Four additional NAD/EDM clinically affected cases were by sires unavailable for phenotyping. A mode of inheritance could not be determined from this small pedigree. When evaluating the full pedigrees on all horses, a common ancestor was found within approximately 15 generations (Fig. S1).

\section{DISCUSSION}

Retinal degeneration is a well-recognized feature of diseases characterized by a-TP deficiencies in many species. ${ }^{3-8}$ The photoreceptor outer segments of the retina are particularly vulnerable to a deficiency of vitE due to a high rate of oxidative metabolism, resulting in increased lipid peroxidation. In addition, more than $65 \%$ of the membrane fatty acids within the rod outer segments are polyunsaturated, leaving this tissue particularly vulnerable to peroxidation. ${ }^{21}$ In dogs with vitE deficiencies and horses with EMND, oxidative damage to the photoreceptors has clearly been documented. ${ }^{5,9}$ Ideally, histologic confirmation of pigment retinopathy at necropsy would have been performed in the present study but unfortunately this was not possible due to processing artifacts within the ocular tissue. Nevertheless, the results of this study indicate that retinal pigmentation is not only a feature of EMND but can also be present in clinically affected NAD/EDM WB horses with a-TP deficiency. Therefore, an ophthalmologic examination is indicated in young horses with proprioceptive ataxia, particularly if they are deficient in a-TP. The presence of pigment within the retina is a useful indicator of an underlying a-TP deficiency, even if there are no associated neurologic abnormalities noted at that time. This finding warrants assessment of serum $\mathrm{a}$-TP concentrations and subsequent supplementation if indicated. The presence of pigment could be associated with vision changes and warrants further electrodiagnostic testing. 
The results of this study contrast the results of a detailed evaluation of the retina in Quarter Horses with NAD that included ERGs. ${ }^{15}$ In this previous study, a pigment retinopathy was not identified in clinically affected NAD/EDM Quarter Horses. ${ }^{15}$ The reasons for the differences in retinal pigmentation between the WBs and the Quarter Horses with NAD/EDM could potentially be the result of genetic differences, the duration, or the severity of a-TP deficiency. The serum a-TP concentrations of the clinical NAD/EDM-affected WBs in our current study were lower (median of all cases $0.89 \mu \mathrm{g} / \mathrm{mL}$, two postmortem confirmed cases 0.41 and $0.42 \mu \mathrm{g} / \mathrm{mL}$ ) than the values obtained from the two of six clinical NAD/EDM Quarter Horses that had serum a-TP concentrations assessed in our previous study $(0.96$ and $1.3 \mu \mathrm{g} / \mathrm{mL}) .{ }^{15}$ Additionally, the grade of ataxia in the four horses with mildto-moderate pigment retinopathy in our current study was worse (grade 2.5 [ $n=2], 3$ [1], and 4 [1]) than the six clinical NAD/EDM horses from the previous study (grade 2 [3], 2.5 [2], 3 [1]). ${ }^{15}$ The duration of a-TP deficiency is less likely to explain the differences in the presence of retinal pigmentopathy between the WBs and Quarter Horses. Quarter Horses with NAD/EDM were similar in age or older $\left(2,5\right.$, and 9 years of age ${ }^{15}$ than the NAD/EDM WB in our current study (range 2-12 years, median 3 years).

It was of particular interest in the present study that both postmortem confirmed cases of NAD/EDM had histopathologic features of both EMND and NAD/EDM. Scattered chromatolytic neurons and spheroids with intraneuronal lipofuscin were noted in the ventral gray column of both NAD/EDM horses at necropsy; lesions that are typical of EMND. ${ }^{20}$ Thus, although the distinction between the two diseases is clinically quite apparent with NAD/EDM characterized by general proprioceptive symmetric ataxia in young horses, and EMND characterized by lower motor neuron weakness and atrophy in older horse, ${ }^{11,17}$ histopathologic lesions can overlap. This is in agreement with a recent report of concurrent NAD/EDM and EMND in three young Paint or Thoroughbred horses that shared a common relative. ${ }^{22}$ In this previous report, while two of these horses resembled EMND and one resembled NAD/EDM on clinical evaluation, histologic lesions consistent with both diseases were observed at postmortem examination. ${ }^{22}$

Six of the NAD/EDM-affected horses, including the two postmortem confirmed concurrent NAD/EDM and EMND horses and four clinically affected NAD/EDM horses, and the clinically affected EMND horses were by one sire. The sire was graded as equivocal on the neurologic evaluation and many of the dams were unavailable for phenotyping. The four clinically affected NAD/EDM horses could be traced back, along the dam lines, to a common ancestor within 15 generations. Without additional phenotypic information on these ancestors, this common ancestor may be purely incidental. As this particular farm had increased the number of horses over time with no available pasture, the impact of an a-TP deficiency on the younger horses may have played a role. While NAD/EDM has been demonstrated to have a strong genetic susceptibility in addition to requiring a temporal a-TP deficiency, EMND has been considered to not have an inherited component. This is the second report of NAD/EDM and EMND occurring in related horses ${ }^{22}$ and a hereditary role to EMND should be considered in these families.

A limitation of this study was that only 2 of 10 NAD/EDM were postmortem confirmed and that the diagnosis of EMND in WB3 was a clinical diagnosis. It remains possible that one or 
more of the ataxic horses could have had another cause for ataxia such as cervical vertebral compressive myelopathy or equine protozoal myeloencephalitis. It is also possible that subclinical or pathologic lesions of EMND were present on other horses on the farm. Further testing was not pursued as the owner had financial constraints and declined additional diagnostic testing. A peripheral nerve or muscle biopsy would have been necessary to confirm our diagnosis of EMND in WB3, however, because serum a-TP concentrations had improved with supplementation, the owner elected to continue supplementation rather than pursue invasive diagnostic testing. Follow-up three months later revealed that the horse was back in training and performing well, although the degree of pigment retinopathy remained unchanged. This is consistent with a diagnosis of EMND as approximately $40 \%$ of horses will respond to a-TP supplementation. ${ }^{17} \mathrm{~A}$ further differential diagnosis for EMND would have been a vitE -deficient myopathy; however, this would have required a muscle biopsy for diagnosis and may be a prodromal stage of EMND. ${ }^{23}$ For the remaining horses, the owner elected to lease pasture in order to provide a source of natural vitE. Recheck a-TP concentrations and neurologic and fundic evaluations were unfortunately not available on the other horses.

In conclusion, clinical and histopathologic findings of NAD/EDM and EMND can occur on the same farm within related individuals in conjunction with a widespread a-TP deficiency. A complete ophthalmologic examination is warranted in suspect horses as a pigment retinopathy may be identified in some horses with clinical NAD/EDM. Pigment retinopathy may also occur in horses with evidence of prolonged a-TP deficiency but without clinical signs of neuromuscular disease. The presence of pigment could be associated with vision changes and may serve as a diagnostic indicator of an underlying a-TP deficiency.

\section{Supplementary Material}

Refer to Web version on PubMed Central for supplementary material.

\section{Acknowledgments}

Dr. Finno's postdoctorate was supported by Morris Animal Foundation (D12EQ-401) and NIH NCATS (K01OD015134-01A1 and L40 TR001136).

\section{References}

1. Schneider C. Chemistry and biology of vitamin E. Molecular Nutrition \& Food Research. 2005; 49:7-30. [PubMed: 15580660]

2. Finno CJ, Valberg SJ. A comparative review of vitamin E and associated equine disorders. Journal of Veterinary Internal Medicine. 2012; 26:1251-1266. [PubMed: 22925200]

3. Yokota T, Igarashi K, Uchihara T, et al. Delayed-onset ataxia in mice lacking alpha-tocopherol transfer protein: model for neuronal degeneration caused by chronic oxidative stress. Proceedings of the National Academy of Sciences of the United States of America. 2001; 98:15185-15190. [PubMed: 11752462]

4. Hayes KC. Pathophysiology of vitamin E deficiency in monkeys. The American Journal of Clinical Nutrition. 1974; 27:1130-1140. [PubMed: 4214211]

5. Riis RC, Sheffy BE, Loew E, et al. Vitamin E deficiency retinopathy in dogs. American Journal of Veterinary Research. 1981; 42:74-86. [PubMed: 7224322] 
6. McLellan GJ, Cappello R, Mayhew IG, et al. Clinical and pathological observations in English cocker spaniels with primary metabolic vitamin E deficiency and retinal pigment epithelial dystrophy. The Veterinary Record. 2003; 153:287-292. [PubMed: 14509574]

7. Davidson MG, Geoly FJ, Gilger BC, et al. Retinal degeneration associated with vitamin E deficiency in hunting dogs. Journal of the American Veterinary Medical Association. 1998; 213:645-651. [PubMed: 9731258]

8. Yokota T, Shiojiri T, Gotoda T, et al. Retinitis pigmentosa and ataxia caused by a mutation in the gene for the alpha-tocopherol-transfer protein. The New England Journal of Medicine. 1996; 335:1770-1771. [PubMed: 8965888]

9. Riis RC, Jackson C, Rebhun W, et al. Ocular manifestations of equine motor neuron disease. Equine Veterinary Journal. 1999; 31:99-110. [PubMed: 10213421]

10. Muller DP. Vitamin E and neurological function. Molecular Nutrition \& Food Research. 2010; 54:710-718. [PubMed: 20183831]

11. Aleman M, Finno CJ, Higgins RJ, et al. Evaluation of epidemiological, clinical, and pathological features of neuroaxonal dystrophy in Quarter Horses. Journal of the American Veterinary Medical Association. 2011; 239:823-833. [PubMed: 21916766]

12. Finno CJ, Higgins RJ, Aleman M, et al. Equine degenerative myeloencephalopathy in Lusitano horses. Journal of Veterinary Internal Medicine. 2011; 25:1439-1446. [PubMed: 22092640]

13. Finno CJ, Estell K, Katzman S, et al. Blood and cerebrospinal fluid alpha-tocopherol and selenium concentrations in neonatal foals with neuroaxonal dystrophy. Journal of Veterinary Internal Medicine. 2015; 29:1667-1675. [PubMed: 26391904]

14. Mohammed HO, Divers TJ, Summers BA, et al. Vitamin E deficiency and risk of equine motor neuron disease. Acta Veterinaria Scandinavica. 2007; 49:17. [PubMed: 17605810]

15. Finno CJ, Aleman M, Ofri R, et al. Electrophysiological studies in American Quarter horses with neuroaxonal dystrophy. Veterinary Ophthalmology. 2012; 15(Suppl 2):3-7. [PubMed: 22432889]

16. Garbe, JR., Da, Y. Pedigraph: A Software Tool for the Graphing and Analysis of Large Complex Pedigrees. User Manual Version 2.4. Department of Animal Science, University of Minnesota, Minneapolis; MN: 2008.

17. Divers TJ, De Lahunta A, Hintz HF, et al. Equine motor neuron disease. Equine Veterinary Education. 2001; 13:63-67.

18. Finno CJ, Famula T, Aleman M, et al. Pedigree analysis and exclusion of alpha-tocopherol transfer protein (TTPA) as a candidate gene for neuroaxonal dystrophy in the American Quarter Horse. Journal of Veterinary Internal Medicine. 2013; 27:177-185. [PubMed: 23186252]

19. Finno CJ, Aleman M, Higgins RJ, et al. Risk of false positive genetic associations in complex traits with underlying population structure: a case study. Veterinary Journal. 2014; 202:543-549.

20. Cummings JF, de Lahunta A, George C, et al. Equine motor neuron disease; a preliminary report. The Cornell Veterinarian. 1990; 80:357-379. [PubMed: 2209016]

21. Stone WL, Farnsworth CC, Dratz EA. A reinvestigation of the fatty acid content of bovine, rat and frog retinal rod outer segments. Experimental Eye Research. 1979; 28:387-397. [PubMed: 446567]

22. Finno CJ, Miller AD, Siso S, et al. Concurrent equine degenerative myeloencephalopathy and equine motor neuron disease in three young horses. Journal of Veterinary Internal Medicine. 2016; 30:1344-50. In Press. [PubMed: 27298214]

23. Bedford HE, Valberg SJ, Firshman AM, et al. Histopathologic findings in the sacrocaudalis dorsalis medialis muscle of horses with vitamin E-responsive muscle atrophy and weakness. Journal of the American Veterinary Medical Association. 2013; 242:1127-1137. [PubMed: 23547678] 


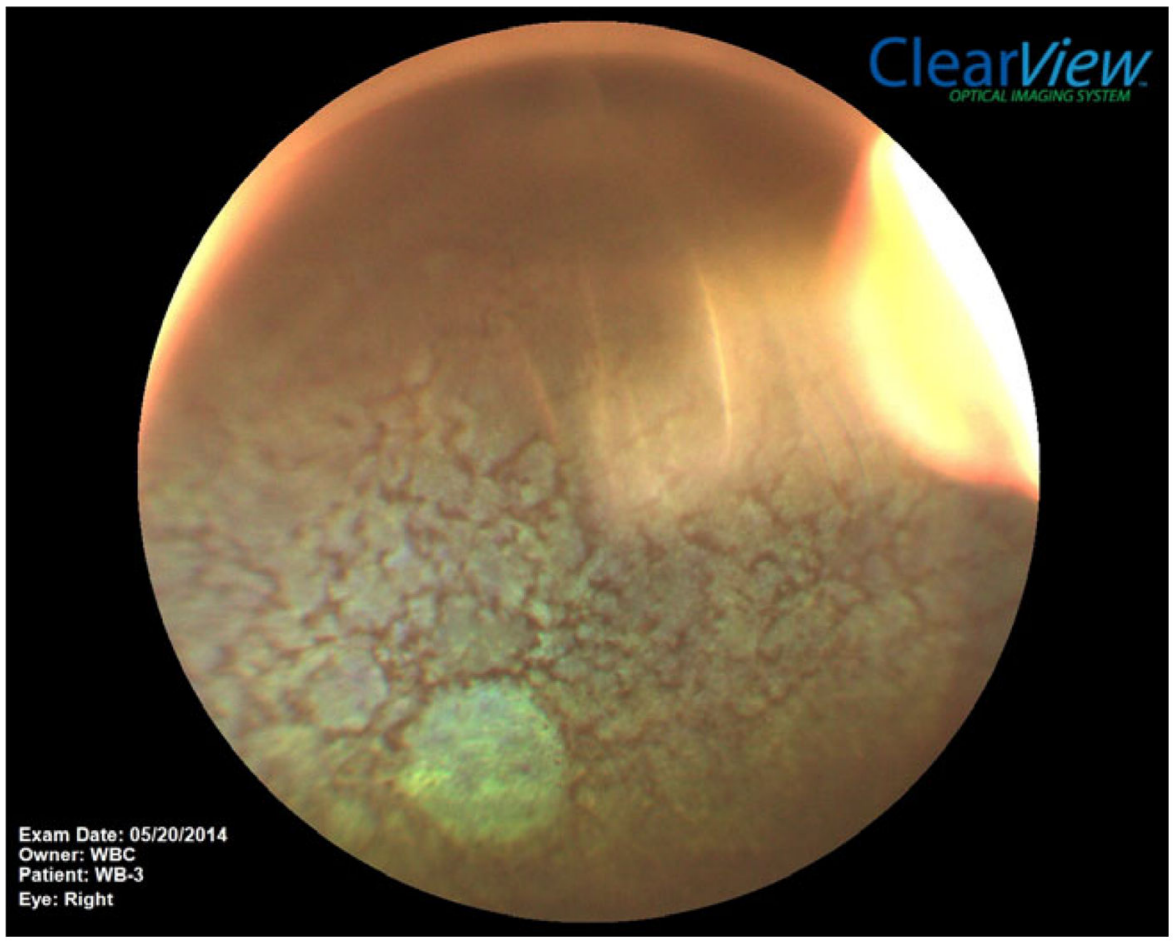

Figure 1.

Tapetal fundus from the right eye of WB3, diagnosed with clinical equine motor neuron disease. Note the distinct reticulated arrangement of pigment through the tapetal zone. 


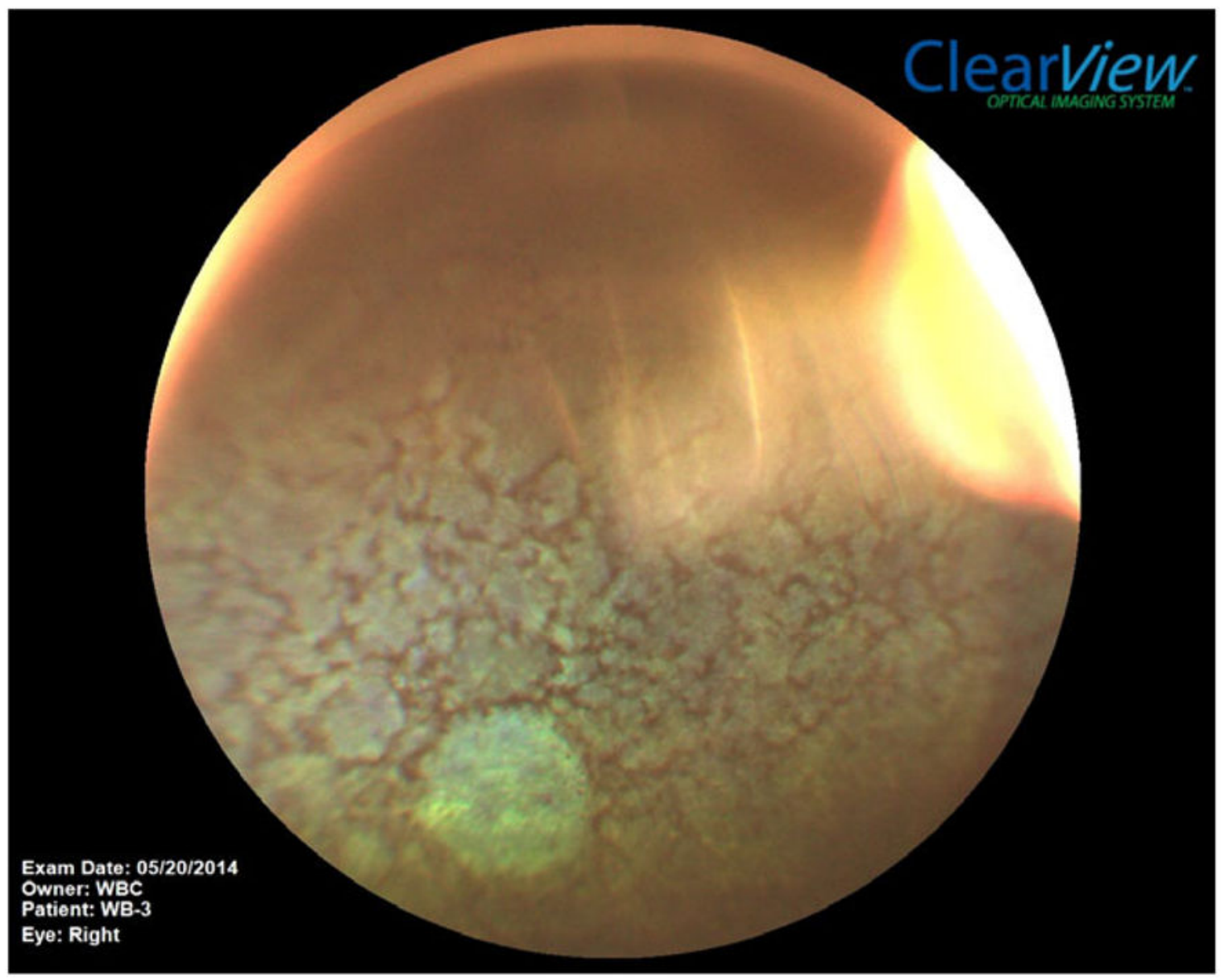

Figure 2.

Tapetal, nontapetal junction from the left eye of WB2, diagnosed with postmortem confirmed concurrent equine degenerative myeloencephalopathy and equine motor neuron disease. Note the pigment bars along the entire tapetal, nontapetal junction with pigment clumping in the tapetal fundus. 


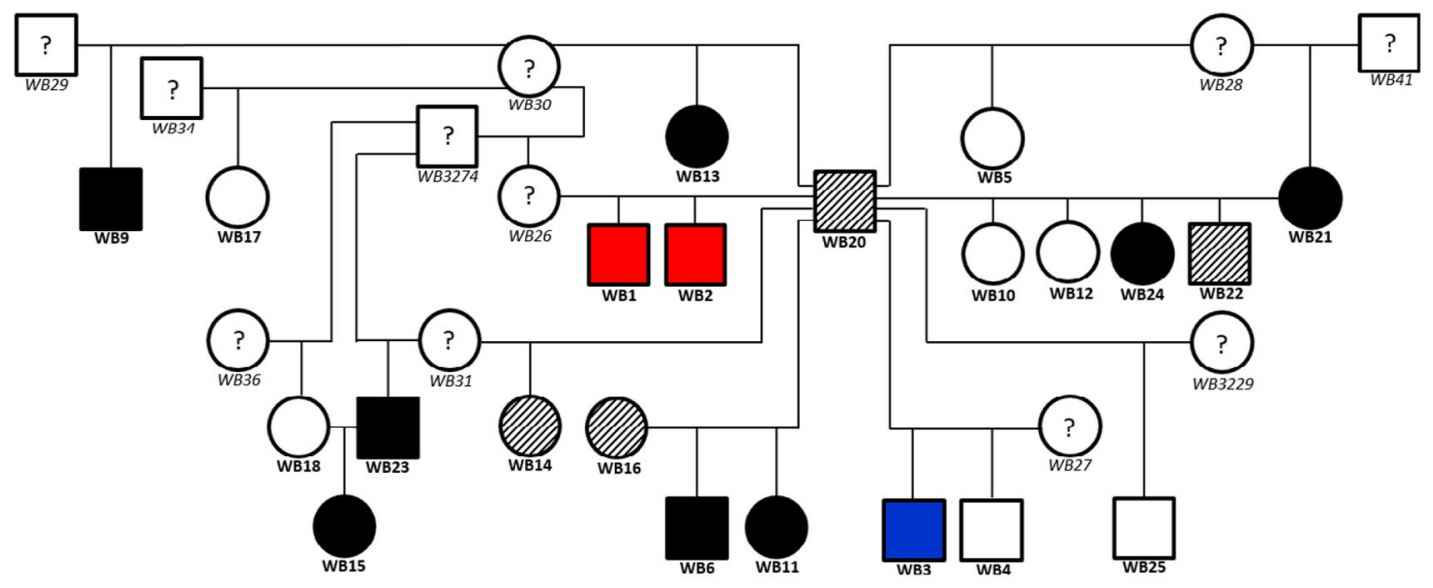

Figure 3.

Pedigree of Warmblood family evaluated for this study. Squares = males, circles $=$ females, red solid $=$ postmortem confirmed neuroaxonal dystrophy/equine degenerative myeloencephalopathy $(\mathrm{NAD} / \mathrm{EDM})$, black solid = clinically diagnosed NAD/EDM-affected horses, blue solid = clinically diagnosed equine motor neuron disease-affected horse, open figure $=$ unaffected, hatched figure $=$ equivocal ataxia score (see Table 1), and $?=$ unavailable for evaluation. Horses evaluated in this study are in bold font and unavailable parents are arbitrarily assigned identification numbers in nonbold italicized font. Horses WB7, WB8, and WB19 are not included in this partial pedigree as they were more distantly related. 


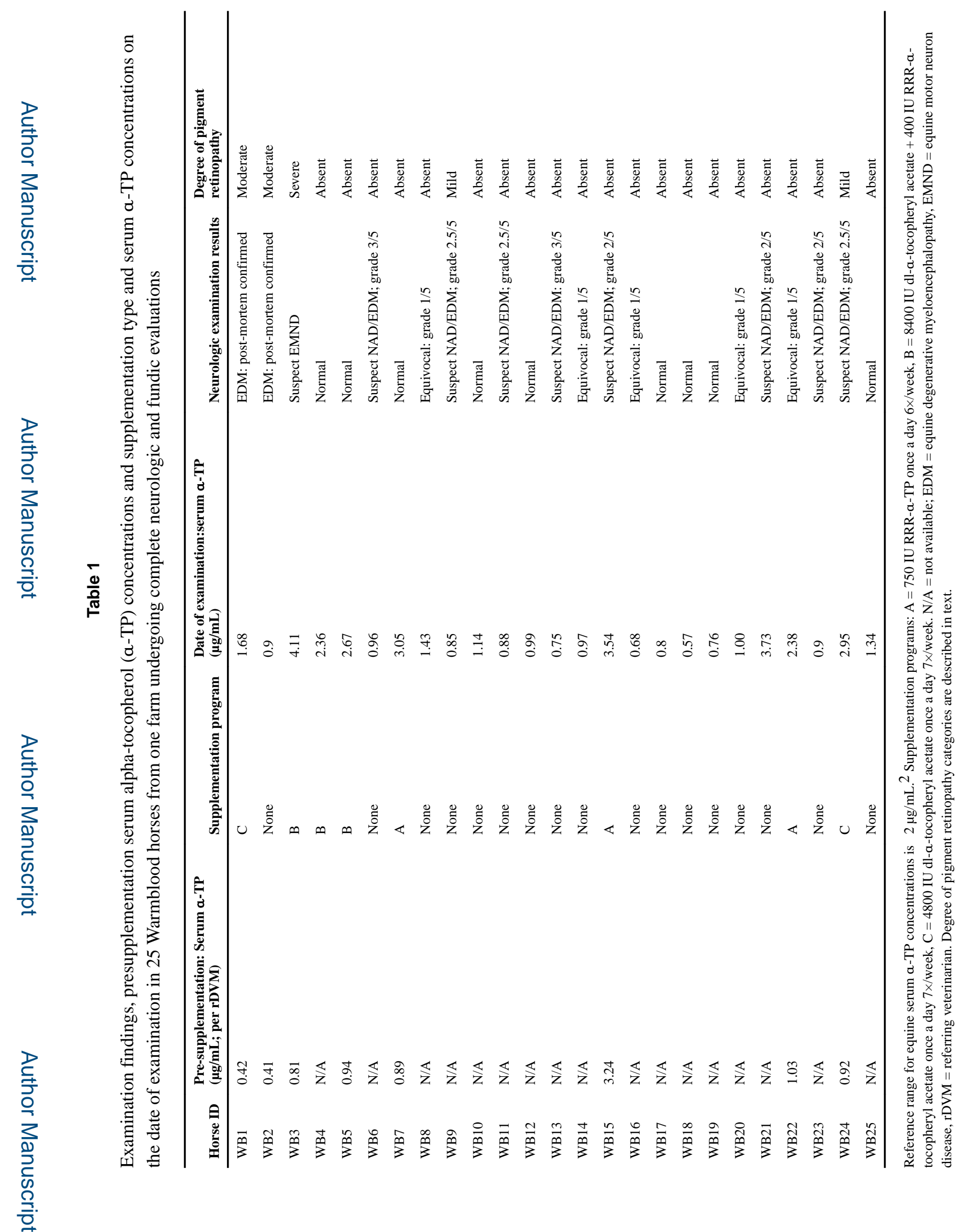

Vet Ophthalmol. Author manuscript; available in PMC 2018 July 01. 